\title{
Linear $L$-positive sets and their polar subspaces
}

\author{
S. Simons \\ Department of Mathematics, University of California \\ Santa Barbara, CA 93106-3080, U.S.A. \\ simons@math.ucsb.edu
}

\begin{abstract}
In this paper, we define a Banach SNL space to be a Banach space with a certain kind of linear map from it into its dual, and we develop the theory of linear $L$-positive subsets of Banach SNL spaces with Banach SNL dual spaces. We use this theory to give simplified proofs of some recent results of Bauschke, Borwein, Wang and Yao, and also of the classical Brezis-Browder theorem.
\end{abstract}

\section{Introduction}

This paper was motivated by a recent result of Bauschke, Borwein, Wang and Yao (which generalizes the Brezis-Browder theorem for reflexive spaces) that if $A$ is a norm-closed linear monotone subspace of the product of a real Banach space with its dual space then the adjoint, $A^{*}$, of $A$ is monotone if, and only if, $A$ is maximally monotone of type (NI). This result appear in Corollary 6.1. We will show in Theorem 5.3 how to obtain relatively simple proofs of generalizations of this results in the context of Banach SNL spaces using the basic tools of convex analysis (that is to say Rockafellar's formula for the subdifferential of a sum, and the Brøndsted-Rockafellar theorem).

We introduce Banach SNL spaces in Section 2, and in Section 3, we give details of how this concept can be applied to monotone subsets of the product of a real Banach space with its dual space. In particular, we show in Lemma 3.4 how the definition of the concept of maximal monotone subset of type (NI) can be recast in a very simple way in the notation of Banach SNL spaces.

In Section 4, we lay out for the benefit of the reader the basic tools of convex analysis (mentioned above) that we will use.

The central section of this paper, Section 5, is about linear $L$-positive sets and their polar subspaces. If $C$ is a convex $L$-positive subset of $B$, we introduce a function $q^{C}$; we show in Lemma 5.1 that this function is convex, and we characterize its subdifferential in Lemma 5.2. Theorem 5.3(a) is the main result in this paper, and Theorem 5.3(b-d) are all fairly simple consequences of Theorem 5.3(a).

In Section 6, we show how to deduce the results on linear monotone subspaces of the product of a real Banach space with its dual that we mentioned above and, in Section 7, we introduce a surjectivity hypothesis that enables us to deduce the Brezis-Browder theorem for reflexive spaces.

In Section 8, we describe an isometry result on Banach SNL spaces with Banach SNL duals that was not needed in the previous sections.

While it is true that the introduction of SNL spaces to solve certain problems on monotone sets does increase the level of abstraction, it is also true that the resulting simplification of the proofs makes it easier to see the essential structure behind them. In particular, the inequality (9) (which appears again in (17)) turns out to be very important. 


\section{Linear $L$-positive sets and their polar subspaces}

The author would like to thank Liangjin Yao for sending him the preprints [3], [4], [5] and [16], and Maicon Marques Alves for sending him the preprint [8].

\section{Banach SNL spaces and Banach SNL duals}

We start off by introducing some Banach space notation.

Definition 2.1. If $X$ is a nonzero real Banach space and $f: X \rightarrow]-\infty, \infty]$, we say that $f$ is proper if there exists $x \in X$ such that $f(x) \in \mathbb{R}$. We write $X^{*}$ for the dual space of $X$ (with the pairing $\langle\cdot, \cdot\rangle: X \times X^{*} \rightarrow \mathbb{R}$ ) and $X^{* *}$ for the bidual of $X$ (with the pairing $\left.\langle\cdot, \cdot\rangle: X^{*} \times X^{* *} \rightarrow \mathbb{R}\right)$. If $x \in X$, we write $\widehat{x}$ for the canonical image of $x$ in $X^{* *}$, that is to say $\quad x \in X$ and $x^{*} \in X^{*} \Longrightarrow\left\langle x^{*}, \widehat{x}\right\rangle=\left\langle x, x^{*}\right\rangle$.

We now introduce the concept of Banach SNL space. Many of the results in this section appear (implicitly) in [12], [13], [14] and [15].

Definition 2.2. Let $B$ be a nonzero real Banach space. A $S N L$ map on $B$ ("SNL" stands for "symmetric nonexpansive linear"), is a linear map $L: B \rightarrow B^{*}$ such that

$$
\|L\| \leq 1 \quad \text { and }, \quad \text { for all } b, c \in B, \quad\langle b, L c\rangle=\langle c, L b\rangle
$$

A Banach SNL space $(B, L)$ is a nonzero real Banach space $B$ together with a SNL map $L: B \rightarrow B^{*}$. We define the function $q_{L}: B \rightarrow \mathbb{R}$ by $q_{L}(b):=\frac{1}{2}\langle b, L b\rangle \quad(b \in B) \quad$ (" $q$ " stands for "quadratic"). Clearly

$$
q_{L}+\frac{1}{2}\|\cdot\|^{2} \geq 0 \text { on } B
$$

It also follows from (1) that, for all $d, e \in B, \quad\left|q_{L}(d)-q_{L}(e)\right|=\frac{1}{2}|\langle d, L d\rangle-\langle e, L e\rangle|=$ $\frac{1}{2}|\langle d-e, L(d+e)\rangle| \leq \frac{1}{2}\|d-e\|\|d+e\|$, and so

$$
q_{L} \text { is continuous. }
$$

Now let $(B, L)$ be a Banach SNL space and $A \subset B$. We say that $A$ is $L$-positive if $A \neq \emptyset$ and $b, c \in A \Longrightarrow q_{L}(b-c) \geq 0$. We say that $A$ is $L$-negative if $A \neq \emptyset$ and $b, c \in A \Longrightarrow q_{L}(b-c) \leq 0$. We say that $A$ is maximally $L$-positive if $A$ is $L$-positive and $A$ is not properly contained in any other $L$-positive set. In this case,

$$
d \in B \Longrightarrow \inf _{a \in A} q_{L}(d-a) \leq 0
$$

(If $d \in B \backslash A$ then the maximality gives us $a \in A$ such that $q_{L}(d-a)<0$, while if $d \in A$ then $q_{L}(d-d)=0$.) Similarly, we say that $A$ is maximally $L$-negative if $A$ is $L$-negative and $A$ is not properly contained in any other $L$-negative set.

Now let $(B, L)$ be a Banach SNL space and $\left(B^{*}, \widetilde{L}\right)$ also be a Banach SNL space. We say that $\left(B^{*}, \widetilde{L}\right)$ is a Banach $S N L$ dual of $(B, L)$ if,

$$
\text { for all } b \in B, \quad \widetilde{L}(L b)=\widehat{b} \text {. }
$$




\section{Linear $L$-positive sets and their polar subspaces}

Then (5) and (1) imply that,

for all $b \in B$ and $b^{*} \in B^{*}, \quad q_{\widetilde{L}}\left(b^{*}+L b\right)=\frac{1}{2}\left\langle b^{*}+L b, \widetilde{L} b^{*}+\widehat{b}\right\rangle=q_{\widetilde{L}}\left(b^{*}\right)+\left\langle b, b^{*}\right\rangle+q_{L}(b)$.

In particular,

$$
q_{\widetilde{L}} \circ L=q_{L}
$$

By analogy with (2), we have

$$
q_{\widetilde{L}}+\frac{1}{2}\|\cdot\|^{2} \geq 0 \text { on } B^{*}
$$

If $(B, L)$ is a Banach SNL space then so also is $(B,-L)$. Further, if $\left(B^{*}, \widetilde{L}\right)$ is a Banach SNL dual of $(B, L)$ then, as the reader can easily verify, $\left(B^{*},-\widetilde{L}\right)$ is a Banach $S N L$ dual of $(B,-L)$.

There are many examples of Banach SNL spaces and their associated $L$-positive sets. The following are derived from [14, Examples 2.3, 2.5, Remarks 6.3, 6.7, 6.8, pp. 230-231, 244-246]. More examples can be found in [7].

Example 2.3. Let $B$ be a Hilbert space with inner product $(b, c) \mapsto\langle b, c\rangle$ and $L: B \rightarrow B$ be a nonexpansive self-adjoint linear operator. Then $(B, L)$ is a Banach SNL space. Here are three special cases of this example:

(a) If, for all $b \in B, L b=b$ then every subset of $B$ is $L$-positive.

(b) If, for all $b \in B, L b=-b$ then the $L$-positive sets are the singletons.

(c) If $B=\mathbb{R}^{3}$ and $L\left(b_{1}, b_{2}, b_{3}\right)=\left(b_{2}, b_{1}, b_{3}\right)$ and $M$ is any nonempty monotone subset of $\mathbb{R} \times \mathbb{R}$ (in the obvious sense) then $M \times \mathbb{R}$ is an $L$-positive subset of $B$. The set $\mathbb{R}(1,-1,2)$ is an $L$-positive subset of $B$ which is not contained in a set $M \times \mathbb{R}$ for any monotone subset of $\mathbb{R} \times \mathbb{R}$. The helix $\{(\cos \theta, \sin \theta, \theta): \theta \in \mathbb{R}\}$ is an $L$-positive subset of $B$, but if $0<\lambda<1$ then the helix $\{(\cos \theta, \sin \theta, \lambda \theta): \theta \in \mathbb{R}\}$ is not.

(d) If $B=\mathbb{R}^{3}$ and $L\left(b_{1}, b_{2}, b_{3}\right)=\left(b_{2}, b_{3}, b_{1}\right)$ then $(B, L)$ cannot be a Banach SNL space, since (1) fails.

In this paper, we are particularly interested in [14, Example 6.5, p. 245], which we will explain in Example 3.1 below.

\section{Connection with monotone sets: type (NI)}

We suppose in this section that $E$ is a nonzero Banach space.

Example 3.1. Let $B:=E \times E^{*}$ and, for all $\left(x, x^{*}\right) \in B,\left\|\left(x, x^{*}\right)\right\|:=\sqrt{\|x\|^{2}+\left\|x^{*}\right\|^{2}}$. We represent $B^{*}$ by $E^{*} \times E^{* *}$, under the pairing $\left\langle\left(x, x^{*}\right),\left(y^{*}, y^{* *}\right)\right\rangle:=\left\langle x, y^{*}\right\rangle+\left\langle x^{*}, y^{* *}\right\rangle$, and define $L: B \rightarrow B^{*}$ by $L\left(x, x^{*}\right):=\left(x^{*}, \widehat{x}\right)$. Then $(B, L)$ is a Banach SNL space and, for all $\left(x, x^{*}\right) \in B, q_{L}\left(x, x^{*}\right)=\frac{1}{2}\left[\left\langle x, x^{*}\right\rangle+\left\langle x, x^{*}\right\rangle\right]=\left\langle x, x^{*}\right\rangle$. If $\left(x, x^{*}\right),\left(y, y^{*}\right) \in B$ then we have $q_{L}\left(\left(x, x^{*}\right)-\left(y, y^{*}\right)\right)=q_{L}\left(x-y, x^{*}-y^{*}\right)=\left\langle x-y, x^{*}-y^{*}\right\rangle$. Thus if $A \subset B$ then $A$ is $L$-positive exactly when $A$ is a nonempty monotone subset of $B$ in the usual sense, and $A$ is maximally $L$-positive exactly when $A$ is a maximally monotone subset of $B$ in the usual sense. We point out that any finite dimensional Banach SNL space of the form 


\section{Linear $L$-positive sets and their polar subspaces}

described here must have even dimension, and that there are many Banach SNL spaces of finite odd dimension with Banach SNL duals. See [14, Remark 6.7, p. 246].

As usual, the dual norm on $B^{*}=E^{*} \times E^{* *}$ is given by $\left\|\left(y^{*}, y^{* *}\right)\right\|:=\sqrt{\left\|y^{*}\right\|^{2}+\left\|y^{* *}\right\|^{2}}$. By analogy with the analysis above, we define $\widetilde{L}: B^{*} \rightarrow B^{* *}$ by $\widetilde{L}\left(y^{*}, y^{* *}\right)=\left(y^{* *}, \widehat{y^{*}}\right)$. Then $\left(B^{*}, \widetilde{L}\right)$ is a Banach SNL space, and, for all $\left(y^{*}, y^{* *}\right) \in B^{*}, q_{\widetilde{L}}\left(y^{*}, y^{* *}\right)=\left\langle y^{*}, y^{* *}\right\rangle$. Now let $\left(x, x^{*}\right) \in B$. Then $\widetilde{L}\left(L\left(x, x^{*}\right)\right)=\widetilde{L}\left(x^{*}, \widehat{x}\right)=\left(\widehat{x}, \widehat{x^{*}}\right)=\left(\widehat{x, x^{*}}\right)$. Thus $(5)$ is satisfied, and so $\left(B^{*}, \widetilde{L}\right)$ is a Banach SNL dual of $(B, L)$.

Finally, let $d^{*}=\left(y^{*}, y^{* *}\right) \in B^{*}$ and $\varepsilon>0$. From the definition of $\left\|y^{* *}\right\|$, there exists $z^{*} \in E^{*}$ such that $\left\|z^{*}\right\| \leq\left\|y^{* *}\right\|$ and $\left\langle z^{*}, y^{* *}\right\rangle \geq\left\|y^{* *}\right\|^{2}-\varepsilon$. Let $d=\left(0, y^{*}+z^{*}\right) \in B$. But then $d^{*}-L d=\left(y^{*}, y^{* *}\right)-\left(y^{*}+z^{*}, 0\right)=\left(-z^{*}, y^{* *}\right) \in B^{*}$. Since

$q_{\widetilde{L}}\left(-z^{*}, y^{* *}\right)+\frac{1}{2}\left\|\left(-z^{*}, y^{* *}\right)\right\|^{2}=-\left\langle z^{*}, y^{* *}\right\rangle+\frac{1}{2}\left(\left\|z^{*}\right\|^{2}+\left\|y^{* *}\right\|^{2}\right) \leq-\left\langle z^{*}, y^{* *}\right\rangle+\left\|y^{* *}\right\|^{2} \leq \varepsilon$,

we have shown that

$$
\text { for all } d^{*} \in B^{*}, \quad \inf _{d \in B}\left[q_{\widetilde{L}}\left(d^{*}-L d\right)+\frac{1}{2}\left\|d^{*}-L d\right\|^{2}\right] \leq 0 .
$$

Remark 3.2. In [14, Definition 6.4, p. 245], we used the terminology " $L(B)$ is $\widetilde{p}$-dense in $B^{*} "$ to describe the inequality (9).

Definition 3.3 first appeared in [11, Definition 10, p. 183]. It was thought at first that this was a weak definition, but it was proved by Marques Alves and Svaiter in [8, Theorem 4.4, pp. 1084-1086], that if $A$ is a maximally monotone subset of $E \times E^{*}$ of type (NI) then $A$ is of "type $(D)$ " (the opposite implication is obviously true). This result was extended in [14, Theorem 9.9(a), pp. 254-255], where it was proved that if $A$ is a maximally monotone subset of $E \times E^{*}$ of type (NI) then $A$ is of "dense type" and "type (ED)". This has a number of consequences, which are detailed in [14, Theorem $9.9(\mathrm{~b}-\mathrm{f})]$ and [14, Theorem 9.10]. Finally, it was proved by Bauschke, Borwein, Wang and Yao in [4, Theorem 3.1] that if $A$ is a maximally monotone subset of $E \times E^{*}$ then $A$ is of type (NI) if, and only if, $A$ is of "Fitzpatrick-Phelps" type.

What distinguishes the definition of "type (NI)" from the definitions of the other classes of maximally monotone sets mentioned above is that it can be recast easily in the language of Banach SNL spaces, as we will see in Lemma 3.4 below.

Definition 3.3. Let $A \subset E \times E^{*}$. We say that $A$ is maximally monotone of type (NI) if $A$ is maximally monotone and,

$$
\text { for all }\left(y^{*}, y^{* *}\right) \in E^{*} \times E^{* *}, \quad \inf _{\left(x, x^{*}\right) \in A}\left\langle y^{*}-x^{*}, y^{* *}-\widehat{x}\right\rangle \leq 0 \text {. }
$$

Here is the promised reformulation of Definition 3.3 in the notation of Banach SNL spaces. We use the conventions introduced in Example 3.1.

Lemma 3.4 Let $A \subset B:=E \times E^{*}$. Then $A$ is maximally monotone of type (NI) if, and only if, $A$ is maximally $L$-positive and, for all $d^{*} \in B^{*}, \inf _{a \in A} q_{\widetilde{L}}\left(d^{*}-L a\right) \leq 0$.

Proof. This is immediate from the formulae for $q_{\widetilde{L}}$ and $L$ given in Example 3.1. 


\section{Linear $L$-positive sets and their polar subspaces}

\section{Standard results from convex analysis}

For the rest of this paper, we suppose that $(B, L)$ is a Banach SNL space and that $\left(B^{*}, \widetilde{L}\right)$ is a Banach SNL dual of $(B, L)$. We will use the following standard notation and results from convex analysis. If $f: B \rightarrow]-\infty, \infty]$ is proper and convex and $b^{*} \in B^{*}$ then the Fenchel conjugate, $f^{*}$, of $f$ is defined by

$$
f^{*}\left(b^{*}\right):=\sup _{B}\left[b^{*}-f\right] .
$$

If, further, $b \in B$ then the subdifferential of $f$ at $b, \partial f(b)$, is the subset of $B^{*}$ defined by

$$
b^{*} \in \partial f(b) \Longleftrightarrow f(b)+f^{*}\left(b^{*}\right)=\left\langle b, b^{*}\right\rangle
$$

We recall (see Rockafellar, [10, Theorem 3(b), pp. 85-86], Zălinescu, [17, Theorem 2.8.7(iii), p. 127], or [13, Theorem 18.1, p. 74]) the following special case of:

Rockafellar's formula for the subdifferential of a sum. Let $f: B \rightarrow]-\infty, \infty]$ be proper and convex. Then

$$
\partial\left(f+\frac{1}{2}\|\cdot\|^{2}\right)=\partial f+J
$$

where $J=\partial\left(\frac{1}{2}\|\cdot\|^{2}\right)$ is characterized by

$$
c^{*} \in J c \Longleftrightarrow\|c\|^{2}=\left\|c^{*}\right\|^{2}=\left\langle c, c^{*}\right\rangle .
$$

$J$ is called the "duality map". Finally, we recall (see Brøndsted-Rockafellar, [6, p. 608], Zălinescu, [17, Theorem 3.1.2, p. 161], or [13, Theorem 18.6, p. 76]) the following special case of the:

Brøndsted-Rockafellar theorem. Let $f: B \rightarrow]-\infty, \infty]$ be proper convex and lower semicontinuous, $\eta>0, b_{0} \in B, b_{0}^{*} \in B^{*}$ and $f\left(b_{0}\right)+f^{*}\left(b_{0}^{*}\right) \leq\left\langle b_{0}, b_{0}^{*}\right\rangle+\eta^{2}$. Then there exist $b_{1} \in B$ and $b_{1}^{*} \in \partial f\left(b_{1}\right)$ such that $\left\|b_{1}-b_{0}\right\| \leq \eta$ and $\left\|b_{1}^{*}-b_{0}^{*}\right\| \leq \eta$.

\section{$5 \quad$ Linear $L$-positive sets}

In this section, we suppose that $A$ is a linear $L$-positive subspace of $B$. If $C$ is a convex $L$-positive subset of $B$, we define the functions $\left.\left.q^{C}, p^{C}: B \rightarrow\right]-\infty, \infty\right]$ by

$$
q^{C}(b)=\left\{\begin{array}{ll}
q_{L}(b) & \text { if } b \in C) ; \\
\infty & (\text { if } b \in B \backslash C),
\end{array} \text { and } \quad p^{C}(b):= \begin{cases}q_{L}(b)+\frac{1}{2}\|b\|^{2} & \text { (if } b \in C) \\
\infty & \text { (if } b \in B \backslash C)\end{cases}\right.
$$

Now fix $c_{0} \in C$. Then, for all $b \in C, q_{L}(b)+\|b\|\left\|c_{0}\right\|+q_{L}\left(c_{0}\right) \geq q_{L}(b)-\left\langle b, L c_{0}\right\rangle+q_{L}\left(c_{0}\right)=$ $q_{L}\left(b-c_{0}\right) \geq 0$ thus, for all $b \in B, p^{C}(b)=q^{C}(b)+\frac{1}{2}\|b\|^{2} \geq-\|b\|\left\|c_{0}\right\|-q_{L}\left(c_{0}\right)+\frac{1}{2}\|b\|^{2}$. Consequently

$$
p^{C}(b) \rightarrow \infty \quad \text { as } \quad\|b\| \rightarrow \infty .
$$

Lemma 5.1 Let $A$ be a linear $L$-positive subspace of $B, d \in B, C:=A-d$ and $d^{*} \in B^{*}$. Then $q^{C}$ is proper and convex. 


\section{Linear $L$-positive sets and their polar subspaces}

Proof. $q^{C}$ is obviously proper. Suppose that $b, c \in C$ and $\left.\lambda \in\right] 0,1[$. Then

$$
\lambda q_{L}(b)+(1-\lambda) q_{L}(c)-q(\lambda b+(1-\lambda) c)=\lambda(1-\lambda) q_{L}(b-c) \geq 0
$$

This implies the convexity of $q^{C}$. (See [13, Lemma 19.7, pp. 80-81].)

We write $A^{0}$ for the linear subspace $\left\{b^{*} \in B^{*}:\langle A, b\rangle=\{0\}\right\}$ of $B^{*} . A^{0}$ is the "polar subspace of $A$ ". The significance of $A^{0}$ lies in the following lemma:

Lemma 5.2 Let $A$ be a linear $L$-positive subspace of $B . d \in B$ and $C:=A-d$. Then

$$
\partial q^{C}(b)= \begin{cases}L b+A^{0} & (\text { if } b \in C) \\ \emptyset & (\text { if } b \in B \backslash C)\end{cases}
$$

Proof. Since it is obvious that $\partial q^{C}(b)=\emptyset$ if $b \in B \backslash C$, it only remains to show that $\partial q^{C}(b)=L b+A^{0}$ if $b \in C$. So suppose that $b \in C$. Then, since $c-b$ runs through $A$ as $c$ runs through $C$ and, from $(1), q_{L}(b)-q_{L}(a+b)=-\langle a, L b\rangle-q_{L}(a)$,

$$
\begin{aligned}
b^{*} \in \partial q^{C}(b) & \Longleftrightarrow q^{C}(b)+\sup _{c \in C}\left[\left\langle c, b^{*}\right\rangle-q_{L}(c)\right] \leq\left\langle b, b^{*}\right\rangle \\
& \Longleftrightarrow \text { for all } c \in C,\left\langle c-b, b^{*}\right\rangle+q_{L}(b)-q_{L}(c) \leq 0 \\
& \Longleftrightarrow \text { for all } a \in A,\left\langle a, b^{*}\right\rangle+q_{L}(b)-q_{L}(a+b) \leq 0 \\
& \Longleftrightarrow \text { for all } a \in A,\left\langle a, b^{*}-L b\right\rangle \leq q_{L}(a) .
\end{aligned}
$$

Since $q_{L}(a) \geq 0$, this is trivially satisfied if $b^{*} \in L b+A^{0}$. On the other hand, if $b^{*} \in \partial q^{C}(b)$ then it follows from the above that, for all $a \in A$ and $\lambda \in \mathbb{R}$,

$$
\lambda\left\langle a, b^{*}-L b\right\rangle=\left\langle\lambda a, b^{*}-L b\right\rangle \leq q_{L}(\lambda a)=\lambda^{2} q_{L}(a),
$$

and, from standard quadratic arguments, $b^{*}-L b \in A^{0}$, that is to say $b^{*} \in L b+A^{0}$.

Theorem 5.3 was suggested by the analysis in Bauschke, Borwein, Wang and Yao $[3$, Theorem 3.1(iii) $\Longrightarrow$ (ii)] and [5, Proposition 3.1]. In contrast to the analysis in these two papers, the only results on convex functions that we use are Rockafellar's formula for the conjugate of a sum, and the Brøndsted-Rockafellar theorem, as outlined in Section 4 above.

Theorem 5.3. Suppose that $A$ be a norm-closed linear $L$-positive subspace of $B$ and $A^{0}$ is $\widetilde{L}$-negative. Then:

(a) Let $d \in B$ and $C:=A-d$. Then $\inf _{C} p^{C} \leq 0$.

(b) Let $d \in B, C:=A-d$ and $b^{*} \in B^{*}$. Then $-\left(q^{C}\right)^{*}\left(b^{*}\right) \leq \frac{1}{2}\left\|b^{*}\right\|^{2}$.

(c) Let $d \in B$ and $d^{*} \in B^{*}$. Then $\inf _{a \in A} q_{\widetilde{L}}\left(d^{*}-L a\right) \leq q_{\widetilde{L}}\left(d^{*}-L d\right)+\frac{1}{2}\left\|d^{*}-L d\right\|^{2}$.

(d) $A$ is maximally $L-$ positive. 


\section{Linear $L$-positive sets and their polar subspaces}

Proof. (a) It is clear from (2) and (12) that there exists $M>0$ such that, writing $C_{M}:=\{b: b \in C,\|b\| \leq M\}, \inf _{C_{M}} p^{C}=\inf _{C} p^{C} \in[0, \infty[$. Now let $\varepsilon>0$ be arbitrary. Choose $\eta>0$ so that $2 M \eta+3 \eta^{2} \leq \varepsilon$, and $b \in C_{M}$ such that $p^{C}(b) \leq \inf _{C_{M}} p^{C}+\eta^{2}=$ $\inf _{C} p^{C}+\eta^{2}=\inf _{B} p^{C}+\eta^{2}$. We have

$$
\|b\| \leq M \quad \text { and } \quad p^{C}(b)+\left(p^{C}\right)^{*}(0)-\langle b, 0\rangle=p^{C}(b)-\inf _{B} p^{C} \leq \eta^{2} .
$$

From $(3), q_{L}$ is continuous. Since $C$ is norm-closed in $B, p^{C}$ is lower semicontinous, and so the Brøndsted-Rockafellar theorem provides us with $c \in B$ and $b^{*} \in \partial p^{C}(c)$ such that $\|c-b\| \leq \eta$ and $\left\|b^{*}\right\| \leq \eta$. From Rockafellar's formula for the subdifferential of a sum, (10), $\partial p^{C}=\partial q^{C}+J$. Combining this with (13) and Lemma 5.2, we have

$\|c\| \leq M+\eta,\left\|b^{*}\right\| \leq \eta, c \in C$ and there exists $c^{*} \in J c$ such that $b^{*} \in L c+A^{0}+c^{*}$.

From (1), (11) and (14), we have

$$
\left\|b^{*}\right\| \leq \eta \quad \text { and } \quad\left\|L c+c^{*}\right\| \leq\|L c\|+\left\|c^{*}\right\| \leq 2\|c\| \leq 2(M+\eta)
$$

and, from (11) and (6), we have

$$
q_{L}(c)+\|c\|^{2}+q_{\widetilde{L}}\left(c^{*}\right)=q_{L}(c)+\left\langle c, c^{*}\right\rangle+q_{\widetilde{L}}\left(c^{*}\right)=q_{\widetilde{L}}\left(L c+c^{*}\right) .
$$

(14) implies that $b^{*}-L c-c^{*} \in A^{0}$. From (11), (8), (16), the $\widetilde{L}$-negativity of $A^{0}$ and (15),

$$
\begin{aligned}
p^{C}(c) & =q_{L}(c)+\frac{1}{2}\|c\|^{2}=q_{L}(c)+\|c\|^{2}-\frac{1}{2}\left\|c^{*}\right\|^{2} \leq q_{L}(c)+\|c\|^{2}+q_{\widetilde{L}}\left(c^{*}\right)=q_{\widetilde{L}}\left(L c+c^{*}\right) \\
& =q_{\widetilde{L}}\left(b^{*}-L c-c^{*}\right)+\left\langle L c+c^{*}, \widetilde{L} b^{*}\right\rangle-q_{\widetilde{L}}\left(b^{*}\right) \leq\left\langle L c+c^{*}, \widetilde{L} b^{*}\right\rangle+\frac{1}{2}\left\|b^{*}\right\|^{2} \\
& \leq\left\|b^{*}\right\|\left\|L c+c^{*}\right\|+\frac{1}{2}\left\|b^{*}\right\|^{2} \leq 2(M+\eta) \eta+\frac{1}{2} \eta^{2}<\varepsilon .
\end{aligned}
$$

Since $\varepsilon>0$ is arbitrary, this completes the proof of (a).

(b) From the definition of Fenchel conjugate (or the Fenchel-Young inequality), for all $c \in C, \quad-\left(q^{C}\right)^{*}\left(b^{*}\right) \leq q^{C}(c)-\left\langle c, b^{*}\right\rangle \leq q^{C}(c)+\|c\|\left\|b^{*}\right\| \leq q^{C}(c)+\frac{1}{2}\|c\|^{2}+\frac{1}{2}\left\|b^{*}\right\|^{2}=$ $p^{C}(c)+\frac{1}{2}\left\|b^{*}\right\|^{2}$, and the result now follows from (a).

(c) Let $C:=A-d$. Using (6), and the definition of $q^{C}$, we have

$$
\begin{aligned}
\inf _{a \in A} q_{\widetilde{L}}\left(d^{*}-L a\right) & =\inf _{a \in A}\left[q_{\widetilde{L}}\left(d^{*}\right)-\left\langle a, d^{*}\right\rangle+q_{L}(a)\right] \\
& =\inf _{c \in C}\left[q_{\widetilde{L}}\left(d^{*}\right)-\left\langle d+c, d^{*}\right\rangle+q_{L}(d+c)\right] \\
& =\inf _{c \in C}\left[q_{\widetilde{L}}\left(d^{*}\right)-\left\langle d, d^{*}\right\rangle-\left\langle c, d^{*}\right\rangle+q_{L}(d)+\langle c, L d\rangle+q_{L}(c)\right] \\
& =\inf _{c \in C}\left[q_{\widetilde{L}}\left(d^{*}-L d\right)-\left\langle c, d^{*}-L d\right\rangle+q_{L}(c)\right] \\
& =q_{\widetilde{L}}\left(d^{*}-L d\right)-\sup _{c \in C}\left[\left\langle c, d^{*}-L d\right\rangle-q^{C}(c)\right] \\
& =q_{\widetilde{L}}\left(d^{*}-L d\right)-\left(q^{C}\right)^{*}\left(d^{*}-L d\right),
\end{aligned}
$$

and the result follows from (b) with $b^{*}:=d^{*}-L c$.

(d) Let $d \in B$ and $A \cup\{d\}$ be $L$-positive. Then, for all $a \in A, q_{L}(a-d) \geq 0$. If now $C:=A-d$ then $q^{C} \geq 0$ on $C$, and so $p^{C}=q^{C}+\frac{1}{2}\|\cdot\|^{2} \geq \frac{1}{2}\|\cdot\|^{2}$ on $C$. Thus (a) implies that $\inf _{C} \frac{1}{2}\|\cdot\|^{2} \leq 0$, from which $0 \in \bar{C}$, which implies in turn that $d \in \bar{A}$. Since $A$ is closed, $d \in A$. This completes the proof that $A$ is maximally $L$-positive. 


\section{Linear $L$-positive sets and their polar subspaces}

Corollary 5.4. Let $A$ be a norm-closed linear $L$-positive subspace of $B$ and

$$
\text { for all } d^{*} \in B^{*}, \quad \inf _{d \in B}\left[q_{\widetilde{L}}\left(d^{*}-L d\right)+\frac{1}{2}\left\|d^{*}-L d\right\|^{2}\right] \leq 0 \text {. }
$$

Then $(18) \Longleftrightarrow(19) \Longleftrightarrow(20) \Longrightarrow(21)$.

$$
A^{0} \text { is } \widetilde{L} \text {-negative. }
$$

$$
\begin{aligned}
& \text { For all } d^{*} \in B^{*}, \inf _{a \in A} q_{\widetilde{L}}\left(d^{*}-L a\right) \leq 0 . \\
& \text { For all } d^{*} \in A^{0}, \inf _{a \in A} q_{\widetilde{L}}\left(d^{*}-L a\right) \leq 0 .
\end{aligned}
$$

$A$ is maximally $L-$ positive.

Proof. It is immediate from Theorem 5.3(c) and (17) that (18) $\Longrightarrow(19)$, and it is obvious that $(19) \Longrightarrow(20)$. If (20) is true then, using (6), for all $d^{*} \in A^{0}$,

$$
q_{\widetilde{L}}\left(d^{*}\right)+\inf _{a \in A} q_{L}(a)=\inf _{a \in A}\left[q_{\widetilde{L}}\left(d^{*}\right)-\left\langle a, d^{*}\right\rangle+q_{L}(a)\right]=\inf _{a \in A} q_{\widetilde{L}}\left(d^{*}-L a\right) \leq 0 .
$$

Since $\inf _{a \in A} q_{L}(a) \geq 0$, it follows that $q_{\widetilde{L}}\left(d^{*}\right) \leq 0$, and the linearity of $A^{0}$ gives (18). Finally, it is immediate from Theorem 5.3(d) that $(18) \Longrightarrow(21)$.

Corollary 5.5. If $A$ is a norm-closed linear $L$-negative subspace of $B$, and $A^{0}$ is $\widetilde{L}_{-}$ positive then $A$ is maximally $L$-negative.

Proof. This result follows by applying Theorem $5.3(\mathrm{~d})$ to $(B,-L)$, since $L$-negativity is equivalent to $(-L)$-positivity and, $\widetilde{L}$-positivity is equivalent to $(-\widetilde{L})$-negativity.

\section{Linear monotone subspaces}

We suppose in this section that $E$ is a nonzero Banach space, and we use the notation of Example 3.1. We define $\rho: E^{*} \times E^{* *} \rightarrow E^{*} \times E^{* *}$ by $\rho\left(x^{*}, x^{* *}\right):=\left(x^{*},-x^{* *}\right)$. Let $\emptyset \neq D \subset E^{*} \times E^{* *}$. Since $q_{\widetilde{L}} \circ \rho=-q_{\widetilde{L}}$,

$$
D \text { is } \widetilde{L} \text {-negative } \Longleftrightarrow \rho(D) \text { is } \widetilde{L} \text {-positive. }
$$

If $A$ is linear subspace of $E \times E^{*}$ then the adjoint subspace, $A^{*}$, of $E^{*} \times E^{* *}$, is defined by: $\left(y^{*}, y^{* *}\right) \in A^{*} \Longleftrightarrow$ for all $\left(a, a^{*}\right) \in A,\left\langle a, y^{*}\right\rangle=\left\langle a^{*}, y^{* *}\right\rangle$. This definition goes back at least to Arens in [1]. Clearly

$$
A^{*}=\rho\left(A^{0}\right) .
$$

The implication $(24) \Longrightarrow(25)$ below was shown by Bauschke, Borwein, Wang and Yao in a two-stage process as follows: It was first shown in [3, Theorem 3.1(iii) $\Longrightarrow$ (ii)] that if $A$ is a maximally monotone linear subspace of $E \times E^{*}$ and (24) is satisfied then $A$ is of type (NI), and it was subsequently shown in [5, Proposition 3.1] that if $A$ is a norm-closed monotone linear subspace of $E \times E^{*}$ and (24) is satisfied then $A$ is maximal. 


\section{Linear $L$-positive sets and their polar subspaces}

Corollary 6.1. Let $A$ be a norm-closed linear monotone subspace of $E \times E^{*}$. Then the four conditions below are equivalent.

$A^{*}$ is monotone.

For all $\left(y^{*}, y^{* *}\right) \in E^{*} \times E^{* *}, \quad \inf _{\left(x, x^{*}\right) \in A}\left\langle y^{*}-x^{*}, y^{* *}-\widehat{x}\right\rangle \leq 0$.

For all $\left(y^{*}, y^{* *}\right) \in A^{0}, \quad \inf _{\left(x, x^{*}\right) \in A}\left\langle y^{*}-x^{*}, y^{* *}-\widehat{x}\right\rangle \leq 0$.

$A$ is maximally monotone of type (NI).

Proof. This is immediate from (23), (22), (9), Corollary 5.4, and Lemma 3.4.

\section{The surjectivity hypothesis and the Brezis-Browder theorem}

In this section, we will consider the implications of the surjectivity hypothesis:

$$
L(B)=B^{*} .
$$

Lemma 7.1. Let $(B, L)$ be a Banach $S N L$ space, $\left(B^{*}, \widetilde{L}\right)$ be a Banach $S N L$ dual of $(B, L)$ and (26) be satisfied. Then (writing. ${ }^{\mathrm{T}}$ for the adjoint of $\cdot$ in the usual sense):

(a) $\left(B^{* *}, L^{\mathrm{TT}}\right)$ is a Banach $S N L$ dual of $\left(B^{*}, \widetilde{L}\right)$.

(b) $B$ is reflexive.

Proof. Clearly, $L^{\mathrm{TT}}: B^{* *} \rightarrow B^{* * *}$ is linear and $\left\|L^{\mathrm{TT}}\right\|=\left\|L^{\mathrm{T}}\right\|=\|L\| \leq 1$. Now let $b^{* *}, c^{* *} \in B^{* *}$. Then $L^{\mathrm{T}} b^{* *}, L^{\mathrm{T}} c^{* *} \in B^{*}$, and (26) provides us with $b, c \in B$ such that $L b=L^{\mathrm{T}} b^{* *} \quad$ and $L c=L^{\mathrm{T}} c^{* *}$. Thus, from (1),

$$
\begin{aligned}
\left\langle b^{* *}, L^{\mathrm{TT}} c^{* *}\right\rangle & =\left\langle L^{\mathrm{T}} b^{* *}, c^{* *}\right\rangle=\left\langle L b, c^{* *}\right\rangle=\left\langle b, L^{\mathrm{T}} c^{* *}\right\rangle=\langle b, L c\rangle \\
=\langle c, L b\rangle & =\left\langle c, L^{\mathrm{T}} b^{* *}\right\rangle=\left\langle L c, b^{* *}\right\rangle=\left\langle L^{\mathrm{T}} c^{* *}, b^{* *}\right\rangle=\left\langle c^{* *}, L^{\mathrm{TT}} b^{* *}\right\rangle .
\end{aligned}
$$

Thus the analog of $(1)$ is satisfied, and so $\left(B^{* *}, L^{\mathrm{TT}}\right)$ is a Banach SNL space. We next show that

$$
\text { for all } c^{*} \in B^{*}, \quad L^{\mathrm{TT}}\left(\widetilde{L} c^{*}\right)=\widehat{c^{*}} \in B^{* * *} .
$$

To this end, let $c^{*} \in B^{*}$, and $b^{* *}$ be an arbitrary element of $B^{* *}$. (26) provides us with $c \in B$ such that $L c=c^{*}$. Then, from $(5), \quad \widetilde{L} c^{*}=\widetilde{L}(L c)=\widehat{c}, \quad$ and so

$$
\begin{aligned}
\left\langle b^{* *}, L^{\mathrm{TT}}\left(\widetilde{L} c^{*}\right)\right\rangle & =\left\langle L^{\mathrm{T}} b^{* *}, \widetilde{L} c^{*}\right\rangle=\left\langle L^{\mathrm{T}} b^{* *}, \widehat{c}\right\rangle \\
& =\left\langle c, L^{\mathrm{T}} b^{* *}\right\rangle=\left\langle L c, b^{* *}\right\rangle=\left\langle c^{*}, b^{* *}\right\rangle .
\end{aligned}
$$

Thus (27) holds. Since (27) is the analog of (5), this completes the proof of (a).

(b) Let $b^{* *} \in B^{* *}$. Since $L^{\mathrm{T}} b^{* *} \in B^{*}$, (26) provides us with $b \in B$ such that $L b=L^{\mathrm{T}} b^{* *}$. Now let $c^{*}$ be an arbitrary element of $B^{*}$. From (26) again, there exists $c \in B$ such that $L c=c^{*}$. Then, from (1),

$$
\left\langle b, c^{*}\right\rangle=\langle b, L c\rangle=\langle c, L b\rangle=\left\langle c, L^{\mathrm{T}} b^{* *}\right\rangle=\left\langle L c, b^{* *}\right\rangle=\left\langle c^{*}, b^{* *}\right\rangle .
$$

Thus $B$ is reflexive. This completes the proof of (b).

Our next result is suggested by Yao, [16, Theorem 2.4, p. 3]. 


\section{Linear $L$-positive sets and their polar subspaces}

Theorem 7.2. Let the surjectivity hypothesis (26) be satisfied, and $A$ be a norm-closed linear $L$-positive subspace of $B$. Then:

(a) $A$ is maximally $L$-positive if, and only if, $A^{0}$ is $\widetilde{L}$-negative.

(b) $A$ is maximally $L$-positive if, and only if, $A^{0}$ is maximally $\widetilde{L}$-negative.

Proof. "If" in (a) is clear from Theorem 5.3(d). Conversely, let us suppose that $A$ is maximally $L$-positive. Let $d^{*} \in B^{*}$. From (26), there exists $d \in B$ such that $L d=d^{*}$. Then, from (7) and (4),

$$
\begin{aligned}
\inf _{a \in A} q_{\widetilde{L}}\left(d^{*}-L a\right) & =\inf _{a \in A} q_{\widetilde{L}}(L d-L a) \\
& =\inf _{a \in A} q_{\widetilde{L}}(L(d-a))=\inf _{a \in A} q_{L}(d-a) \leq 0
\end{aligned}
$$

and, since (26) implies (17), it follows from Corollary 5.4 that $A^{0}$ is $\widetilde{L}$-negative. This completes the proof of (a). "If" in (b) is immediate from "If" in (a). Conversely, let us suppose that $A$ is maximally $L$-positive. Now $A^{0}$ is norm-closed and, from (a), $\widetilde{L}$-negative, and it only remains to prove the $\widetilde{L}$-maximality. We now show that

$$
\text { for all } a^{* *} \in\left(A^{0}\right)^{0}, \quad q_{L^{\mathrm{TT}}}\left(a^{* *}\right) \geq 0 .
$$

To the end, let $a^{* *} \in\left(A^{0}\right)^{0}$. Since $A$ is norm-closed, standard functional analysis and the reflexivity of $B$ (Lemma $7.1(\mathrm{~b}))$ imply that there exists $a \in A$ such that $a^{* *}=\widehat{a}$. From (5), Lemma $7.1(\mathrm{a})$, and two applications of $(7)$ (one to $(B, L)$ and one to $\left.\left(B^{*}, \widetilde{L}\right)\right)$,

$$
q_{L^{\mathrm{TT}}}\left(a^{* *}\right)=q_{L^{\mathrm{TT}}}(\widehat{a})=q_{L^{\mathrm{TT}}}(\widetilde{L}(L a))=q_{\widetilde{L}}(L a)=q_{L}(a) \geq 0 .
$$

Thus (28) is true, from which it follows that $\left(A^{0}\right)^{0}$ is $L^{\mathrm{TT}}$-positive. If we now apply Corollary 5.5, with $B$ replaced by $B^{*}$, and $A$ replaced by $A^{0}$, we see that $A^{0}$ is maximally $\widetilde{L}$-negative, which completes the proof of $(\mathrm{b})$.

Corollary 7.3(a) below appears in Brezis-Browder [2, Theorem 2, pp. 32-33], and Corollary 7.3(b) in Yao, [16, Theorem 2.4, p. 3].

Corollary 7.3. Let $E$ be reflexive and $A$ be a norm-closed linear monotone subspace of $E \times E^{*}$. Then:

(a) $A$ is maximally monotone if, and only if, $A^{*}$ is monotone

(b) $A$ is maximally monotone if, and only if, $A^{*}$ is maximally monotone.

Proof. These results follow from Theorem 7.2 and the comments in Example 3.1.

\section{The isometry lemma}

In this section, we make an observation about Banach SNL spaces that was not needed in the preceding sections.

Lemma 8.1. Let $(B, L)$ be a Banach $S N L$ space with Banach $S N L$ dual $\left(B^{*}, \widetilde{L}\right)$. Then $L$ is an isometry of $B$ into $B^{*}$. 


\section{Linear $L$-positive sets and their polar subspaces}

Proof. Since $\|L\| \leq 1$ and $\|\widetilde{L}\| \leq 1$, for all $b \in B$,

$$
\|b\|=\|\widehat{b}\|=\|\widetilde{L}(L b)\| \leq\|\widetilde{L}\|\|L b\| \leq\|L b\| \leq\|L\|\|b\| \leq\|b\| .
$$

Thus $\|L b\|=\|b\|$.

Lemma 8.1 implies that if the surjectivity hypothesis, (26), is satisfied then $B$ is what was called a SSDB space in [13, Definition 21.1], [9, Section 1], [15, Definition 4.1] and [7, Section 3]. It also implies that $(\mathbb{R}, 0)$ is a Banach SNL space without a Banach SNL dual.

\section{References}

[1] R. Arens, Operational calculus of linear relations, Pacific J. Math. 11 (1961) 9-23.

[2] H. Brezis and F. E. Browder, Linear maximal monotone operators and singular nonlinear integral equations of Hammerstein, type, Nonlinear analysis (collection of papers in honor of Erich H. Rothe), pp. 31-42. Academic Press, New York, 1978.

[3] H. Bauschke, J. M. Borwein, X. Wang and L. Yao For maximally monotone linear relations, dense type, negative-infimum type, and Fitzpatrick-Phelps type all coincide with monotonicity of the adjoint, http://arxiv.org/abs/1103.6239v1, posted March 31, 2011.

[4] H. Bauschke, J. M. Borwein, X. Wang and L. Yao Every maximally monotone operator of Fitzpatrick-Phelps type is actually of dense type, http://arxiv.org/abs/1104.0750, posted April 5, 2011.

[5] H. Bauschke, J. M. Borwein, X. Wang and L. Yao The Brezis-Browder Theorem in a general Banach space, http://arxiv.org/abs/1110.5706v1, posted October 26, 2011.

[6] A. Brøndsted and R.T. Rockafellar, On the Subdifferentiability of Convex Functions, Proc. Amer. Math. Soc. 16(1965), 605-611.

[7] Y. García Ramos, J. E. Martínez-Legaz and S. Simons, New results on q-positivity, http://arxiv.org/abs/1111.6094v1, posted November 25, 2011.

[8] M. Marques Alves and B. F. Svaiter, On Gossez type (D) maximal monotone operators., J. of Convex Anal., 17 (2010), 1077-1088.

[9] J. E. Martínez-Legaz, On maximally q-positive sets, J. of Convex Anal., 16 (2009), 891-898.

[10] R. T. Rockafellar, Extension of Fenchel's duality theorem for convex functions, Duke Math. J. 33 (1966), 81-89.

[11] S. Simons, The range of a monotone operator, J. Math. Anal. Appl. 199 (1996), 176-201.

[12] — - Positive sets and Monotone sets, J. of Convex Anal., 14 (2007), 297-317.

[13] —-, From Hahn-Banach to monotonicity, Lecture Notes in Mathematics, 1693, second edition, (2008), Springer-Verlag.

[14] — B Banach SSD spaces and classes of monotone sets, J. of Convex Anal., 18 (2011), $227-258$.

[15] — - SSDB spaces and maximal monotonicity, J. Glob. Optim., 50 (2011), 23-57.

[16] L. Yao, The Brezis-Browder theorem revisited and properties of Fitzpatrick functions of order n, http://arxiv.org/abs/0905.4056v1, posted May 25, 2009.

[17] C. Zălinescu, Convex analysis in general vector spaces, (2002), World Scientific. 